\title{
Erratum: Increased Defect Size is Associated with Increased Complication Rate after Free Tissue Transfer for Midanterior Skull-Base Reconstruction
}

\author{
Ryota Nakamura ${ }^{1,2}$ Keisuke Takanari ${ }^{2}$ Yutaka Nakamura ${ }^{2}$ Miki Kambe ${ }^{2}$ Masashi Hishida ${ }^{3}$ \\ Katsumi Ebisawa ${ }^{2}$ Yasushi Fujimoto ${ }^{4}$ Masazumi Fujii ${ }^{5}$ Yuzuru Kamei ${ }^{2}$
}

${ }^{1}$ Department of Plastic and Reconstructive Surgery, Aichi Cancer Center, Nagoya, Aichi, Japan

2 Department of Plastic and Reconstructive Surgery, Nagoya

University Graduate School of Medicine, Nagoya, Japan

${ }^{3}$ Department of Plastic and Reconstructive surgery, Japanese Red Cross Nagoya Daiichi Hospital, Nagoya, Japan

${ }^{4}$ Department of Otorhinolaryngology, Nagoya University Graduate School of Medicine, Nagoya, Japan

${ }^{5}$ Department of Neurosurgery, Fukushima Medical University, Fukushima, Japan

J Neurol Surg B 2020;81:e1.
Address for correspondence Ryota Nakamura, MD, Department of Plastic and Reconstructive Surgery, Aichi Cancer Center, 1-1 kanokoden, Chikusa-ku, Nagoya, Aichi 464-8681, Japan (e-mail: rnakamura@aichi-cc.jp).

\section{ERRATUM}

It has been brought to our attention that the order of author names appeared incorrectly in the above article published online in Journal of Neurological Surgery Part B (DOI: 10.1055/s-00381676777). The order has now been corrected as shown above. 